\title{
Methods for Generation of Spatial Gradients in Concentration of Monomeric Surfactants and Micelles in Microfluidic Systems
}

Xiaoyang Liu, Michael D. Graham and Nicholas L. Abbott ${ }^{*}$

Department of Chemical and Biological Engineering, University of Wisconsin, 1415

Engineering Drive, Madison, WI 53705-1691

\section{Supplemental Materials}

When the surfactant concentration is above the CMC of reduced FTMA, the following equations can be derived from Equation (3) based on the fact that the mass flux of oxidized FTMA, reduced FTMA and micelles are equal to each other at steady-state:

$$
\begin{gathered}
c_{O}=A x+B \\
c_{R}=\left\{\begin{array}{l}
-A x+F\left(0 \leq x \leq x_{L}\right) \\
-\frac{D_{O}}{D_{M}} A x+G\left(x_{L} \leq x \leq L\right)
\end{array}\right.
\end{gathered}
$$

where the subscripts $\mathrm{O}, \mathrm{R}, \mathrm{M}$ indicate oxidized FTMA, reduced FTMA (including monomers and micelles) and micelles respectively, $x_{L}$ is the value of $\mathrm{x}$ at which micelles form, and A, B, F and G are constants to be determined. These two equations contain five constants, which are solved using the following relationships:

$$
\begin{array}{r}
-A x_{L}+F=c_{C M C} \\
-\frac{D_{O}}{D_{M}} A x_{L}+G=c_{C M C} \\
E_{A}=E^{0}+\frac{R T}{n F} \ln \frac{C_{O}}{C_{R}}
\end{array}
$$




$$
\begin{gathered}
E_{C}=E^{0}+\frac{R T}{n F} \ln \frac{C_{O}}{C_{R}} \\
\int_{0}^{L} c_{O} d x+\int_{0}^{x_{L}} c_{R} d x+\int_{x_{L}}^{L} c_{R} d x=c^{*} L
\end{gathered}
$$

Equations 9 and 10 are based on the fact that the concentration of reduced FTMA at $x_{L}$ is equal to the critical micelle concentration $\left(c_{C M C}\right.$ is the critical micelle concentration of FTMA). Equations 11 and 12 are the Nernst equation applied at the anode and cathode, where $E_{A}$ and $E_{C}$ are potentials of anode and cathode, respectively. Equation 13 is based on mass conservation, where $\mathrm{L}$ is the width of the cell (in $\mathrm{x}$ direction) and $c^{*}$ is the initial concentration of FTMA. 
